\title{
Corrélations phénotype-génotype dans les titinopathies
}

\author{
Raul Juntas-Morales ${ }^{1,2}$, Aurélien Perrin ${ }^{1}$, Mireille Cossée $^{1}$
}

Les pathologies dues à des variants pathogènes du gène TTN codant la titine ou titinopathies représentent un groupe important et très hétérogène de maladies du muscle squelettique. Ce même gène TTN est par ailleurs considéré aujourd'hui comme le principal gène impliqué dans les cardiomyopathies dilatées avec une prévalence de $17 \%$ [1].

Les deux premiers phénotypes de titinopathies à avoir été décrits étaient de transmission autosomique dominante : la dystrophie musculaire tibiale (TMD) et la myopathie héréditaire avec atteinte respiratoire précoce (HMERF). Étant donné que seule la région 3' du gène était initialement étudiée avec les techniques classiques de séquençage, tous les variants pathogènes responsables de ces premiers phénotypes étaient jusqu'ici localisés dans cette région.

Grâce à l'émergence du séquençage à haut débit (NGS) et à une analyse exhaustive des 364 exons du gène, il a été possible d'identifier de nouveaux phénotypes et de confirmer l'existence de variants pathogènes répartis dans d'autres régions du gène.

\section{Premières descriptions}

Dystrophie musculaire tibiale (TMD)

Raul Juntas Morales Laboratoire de Génétique Moléculaire, EA7402,

Centre Hospitalier

Universitaire de

Montpellier, Université de

Montpellier, France

Service de Neurologie.

Centre de référence des

Maladies

Neuromusculaires AOC

(Atlantique-Occitanie-

Caraibe) Centre

Hospitalier Universitaire

de Montpellier, France

Aurélien Perrin,

Mireille Cossée

Laboratoire de Génétique

Moléculaire, EA7402,

Centre Hospitalier

Universitaire de

Montpellier, Université de

Montpellier, France

Contact

r-juntasmorales@

chu-montpellier.fr
La TMD est une myopathie distale dominante d'apparition tardive rapportée initialement de manière exclusive en Finlande du fait d'un effet fondateur. Dans ce pays, elle représente la forme la plus fréquente de myopathie chez les adultes [2]. Le déficit moteur reste très localisé au niveau des muscles releveurs des pieds et l'évolution de la pathologie est très lente. La biopsie musculaire, généralement peu altérée, montre habituellement de la fibrose et des vacuoles bordées [2]. En 2002, une délétion/insertion de $11 \mathrm{pb}$ dans le dernier exon du gène (exon 364), a été identifiée chez tous les patients finlandais diagnostiqués (mutation FINmaj). La conséquence au niveau de la protéine est une substitution de quatre acides aminés [3]. Depuis, d'autres variants pathogènes, majoritairement dans l'exon 364 (mais aussi dans le 363), ont été identifiés par la suite chez des familles originaires d'autres pays européens présentant le même phénotype [4-6].

Plus récemment, des formes de dystrophie musculaire tibiale de transmission autosomique récessive (AR), d'apparition plus précoce et d'évolution plus rapide, ont été décrites chez des patients hétérozygotes composés portant le variant FINmaj associé à un deuxième variant [9]. Par ailleurs, en 2017, une étude a rapporté une série de 14 patients d'origine serbe avec un phénotype de myopathie distale porteurs du même variant non-sens (c.107635C>T; p.Gln35879*) dans l'exon 363 du gène TTN (avec un effet fondateur probable dans cette population). Trois patients étaient homozygotes et les autres étaient hétérozygotes composés avec un deuxième variant localisé, dans la plupart des cas, dans la région codant la bande $\mathrm{M}$ [10].

Par ailleurs, un phénotype sévère de myopathie des ceintures a été décrit (LGMD de type R10 ou anciennement 2), chez des patients finlandais porteurs de la mutation FINmaj à l'état homozygote. Hors de Finlande, des phénotypes similaires ont été décrits. Cependant le variant FINmaj à l'état hétérozygote était associé à un second variant tronquant (une famille française et une autre chinoise) [11-13].

Myopathie héréditaire avec atteinte respiratoire précoce (HMERF)

La myopathie HMERF débute habituellement dans la première ou deuxième décennie de vie par un déficit distal des membres inférieurs s'étendant par la suite aux muscles proximaux. Un syndrome restrictif respiratoire grave se développe systématiquement de manière précoce dans l'évolution de cette pathologie. La biopsie montre typiquement la présence de corps cytoplasmiques. Initialement, la plupart des patients partageaient le même variant faux-sens dans l'exon 344 (c.95134T>C ; p.Cys31712Arg) [7]. Le phénotype clinique a été élargi ces dernières années avec un début plus tardif des symptômes (cinquième ou sixième décennie) et une atteinte cardiaque qui peut survenir pendant l'évolution. Sur le plan génétique, d'autres variants faux-sens ont été rapportés, également dans l'exon 344 [8]. 


\section{Élargissement du spectre clinique des titinopathies}

Titinopathie de type " Emery-Dreifuss-like " Cette titinopathie a été décrite chez des patients présentant un phénotype de myopathie rétractile d'apparition précoce. Les rétractions étaient diffuses mais prédominaient au niveau des biceps brachiaux. Une atteinte respiratoire était associée chez tous les patients. Tous les variants étaient localisés dans la région du gène codant la bande M [14].

Par ailleurs, plusieurs phénotypes différents de myopathie congénitale ont été rapportés : la myopathie précoce avec cardiomyopathie dilatée fatale et la myopathie à multiminicores avec atteinte cardiaque ont été décrites notamment par l'équipe d'Ana Ferreiro à Paris, et plus récemment, des phénotypes de type arthrogrypose multiplex congenita et myopathie de type centronucléaire ont été rapportés [15-18] Tableau I).

Myopathie précoce avec cardiomyopathie dilatée fatale

La myopathie précoce avec cardiomyopathie dilatée fatale a été décrite chez 5 patients issus de deux familles consanguines qui présentaient un déficit musculaire modéré accompagné d'une pseudohypertrophie des mollets, d'un ptosis bilatéral et d'une parésie faciale. La cardiomyopathie, d'apparition précoce, a conduit au décès des patients avant l'âge de 20 ans. Deux variants homozygotes et décalant le cadre de lecture (frameshift) ont été identifiés dans les exons 359 et 361 . Ces variants provoquent la perte de la partie C-terminale de la protéine et notamment du site de liaison à la calpaïne 3 , dans l'exon 363, ce qui explique l'absence de cette dernière dans les études par western-blot (WB) réalisées sur les biopsies musculaires de tous les patients étudiés [15].

Myopathie congénitale à multiminicores avec cardiomyopathie

La myopathie à multiminicores avec atteinte cardiaque a été initialement rapportée chez cinq patients appartenant à quatre familles différentes. Le phénotype clinique et histologique était celui d'une myopathie à multiminicores avec la présence de rétractions au niveau du rachis (rigid spine). La particularité était la présence d'une cardiomyopathie, qui n'est habituellement pas présente dans les formes classiques de myopathie à multiminicores secondaires à des variants des gènes SEPN1 et $R Y R 1$. Sur le plan génétique, deux familles portaient un variant tronquant homozygote situé dans la région codant la bande M (exons 359 et 360) et les deux autres avaient un variant tronquant et un faux-sens en trans. Le variant faux-sens c. 102439T>C ; p.Trp34072Arg d'une des familles était localisé dans le domaine kinase affectant un résidu très conservé et le variant faux-sens c.66920T>A ; p.Val22232Glu de la deuxième famille était situé dans l'exon 316, à la fin de la bande I, domaine de type fibronectine (Fn3) [16]. Ces variations faux-sens pourraient altérer la stabilité des domaines protéiques impactés mais également l'interaction avec d'autres protéines musculaires.

\section{Myopathie de type centronucléaire}

Cinq patients avec un phénotype différent et des anomalies histologiques évocatrices d'une myopathie de type " centronucléaire " avaient été rapportés en 2013 [17]. Cliniquement, ils présentaient un déficit moteur modéré à prédominance axiale et un syndrome restrictif respiratoire. Aucun patient n'avait développé de cardiomyopathie. Sur le plan histologique, un noyau central était visible dans 65 à $85 \%$ des fibres musculaires, raison pour laquelle le diagnostic initialement suspecté était celui d'une myopathie centronucléaire. Tous les patients portaient deux variants TTN prédits pour décaler le cadre de lecture. Cependant, à la différence des autres formes de titinopathie congénitale, les variants étaient situés sur différentes régions du gène et aucun n'était localisé dans la bande M [17].

Dans le but de mieux caractériser l'histoire naturelle et le phénotype clinique et histologique des myopathies congénitales liées à des variants TTN, une cohorte internationale de 30 patients impliquant plusieurs centres experts dans le domaine a été analysée et publiée en 2018 [20]. Seuls les patients porteurs de deux variants tronquants avec décalage du cadre de lecture avaient été inclus dans la cohorte. Du point de vue clinique, il existait une atteinte axiale et des membres de sévérité légère à modérée dans la plupart des cas, accompagnée de rétractions musculaires des membres et paravertébrales. Un ptosis uni ou bilatéral, une discrète parésie faciale et un palais ogival étaient souvent présents. Un syndrome restrictif respiratoire était retrouvé dans environ $2 / 3$ des cas. L'atteinte cardiaque était également fréquente. Sur le plan histologique, les biopsies montraient principalement une combinaison de trois types d'anomalies : internalisations nucléaires, anomalies de la taille des fibres, et minicores. Dans environ un tiers des cas, le diagnostic anatomopathologique retenu initialement était celui d'une myopathie centronucléaire. Sur le plan génétique, les différents variants étaient distribués tout le long du 


\begin{tabular}{|c|c|c|c|c|c|}
\hline NOM & TRANSMISSION & $\begin{array}{l}\text { ÂGE DE } \\
\text { DÉBUT }\end{array}$ & PHÉNOTYPE & $\begin{array}{c}\text { BIOPSIE } \\
\text { MUSCULAIRE }\end{array}$ & VARIANTS GÉNÉTIQUES \\
\hline $\begin{array}{l}\text { Dystrophie musculaire } \\
\text { tibiale (TMD) }\end{array}$ & $\begin{array}{l}\mathrm{AD} \\
\text { Population finlandaise }\end{array}$ & $>35$ ans & $\begin{array}{l}\text { Déficit localisé des } \\
\text { releveurs des pieds }\end{array}$ & $\begin{array}{l}\text { Peu altérée } \\
\text { Fibrose } \\
\text { Vacuoles bordées }\end{array}$ & $\begin{array}{l}\text { - Effet fondateur en Finlande : } \\
11 \text { bp indel dans le dernier exon du } \\
\text { gène : } 364 \text { (Mutation FINMaj) [2] } \\
\text { - En dehors de la Finlande } \\
\text { mutations des exons } 363 \text { et } 364 \\
\text { [4][5][6]. }\end{array}$ \\
\hline $\begin{array}{l}\text { Dystrophie musculaire } \\
\text { tibiale à début précoce }\end{array}$ & $\begin{array}{l}\text { AR } \\
\text { Finlande } \\
\text { Italie, Espagne } \\
\text { Serbie }\end{array}$ & 20 ans & $\begin{array}{l}\text { Déficit localisé des } \\
\text { releveurs des pieds }\end{array}$ & Aspect dystrophique & $\begin{array}{l}\text { - Variant FInMaj associé à un autre } \\
\text { variant [9] } \\
\text { - Variant c. } 107635 \mathrm{C}>\mathrm{T} \text {; } \\
\left.\text { p.(Gln } 35879^{*}\right) \text { dans l'exon } 363 \\
\text { dans la population serbe associé à } \\
\text { un autre variant. }\end{array}$ \\
\hline $\begin{array}{l}\text { Myopathie des } \\
\text { ceintures } \\
\text { LGMDR10/LGMD2J }\end{array}$ & $\begin{array}{l}\text { AR } \\
\text { Finlande } \\
\text { Rares cas ailleurs }\end{array}$ & $<20$ ans & $\begin{array}{l}\text { Déficit proximal } \\
\text { ceintures pelvienne et } \\
\text { scapulaire } \\
\text { Très évolutive }\end{array}$ & Vacuoles bordées & $\begin{array}{l}\text { - Patients finlandais avec le variant } \\
\text { FINmaj homozygote [21] ou } \\
\text { hétérozygote composite avec un } \\
\text { autre variant tronquant [9] } \\
\text { - Une famille française avec un } \\
\text { variant non-sens homozygote dans } \\
\text { l'exon } 364 \text { [12]. Une famille } \\
\text { chinoise avec un variant faux-sens } \\
\text { homozygote dans l'exon } 364 \text { [13]. }\end{array}$ \\
\hline $\begin{array}{l}\text { Myopathie héréditaire } \\
\text { avec atteinte } \\
\text { respiratoire précoce } \\
\text { (HMERF) }\end{array}$ & $\begin{array}{l}\mathrm{AD} \\
\text { Rares cas } \mathrm{AR}\end{array}$ & $>20$ & $\begin{array}{l}\text { Atteinte distale puis } \\
\text { proximo-distale } \\
\text { Très évolutive } \\
\text { Atteinte respiratoire } \\
\text { précoce }\end{array}$ & $\begin{array}{l}\text { Corps cytoplasmiques } \\
\text { Vacuoles bordées }\end{array}$ & $\begin{array}{l}\text { - Variant majoritaire : } \\
\text { c. } 95134 T>C \text {; p.(Cys31712Arg) } \\
\text { dans l'exon } 344 \text { [7] } \\
\text { - Plus rares, d'autres variants } \\
\text { faux-sens dans l'exon } 344 \text { [8] }\end{array}$ \\
\hline $\begin{array}{l}\text { Myopathie Emery } \\
\text { Dreifuss-like }\end{array}$ & AR & $<10$ ans & $\begin{array}{l}\text { Déficit des ceintures } \\
\text { Rétractions d'apparition } \\
\text { précoce } \\
\text { "rigid Spine" } \\
\text { Atteinte respiratoire }\end{array}$ & $\begin{array}{l}\text { Corps cytoplasmiques } \\
\text { Vacuoles bordées } \\
\text { Absence de minicores }\end{array}$ & $\begin{array}{l}\text { - Variants décalant le cadre de } \\
\text { lecture dans les } 3 \text { derniers exons du } \\
\text { gène [14] }\end{array}$ \\
\hline $\begin{array}{l}\text { Myopathie précoce } \\
\text { avec cardiopathie } \\
\text { létale (EOMFC) }\end{array}$ & AR & Néonatale & $\begin{array}{l}\text { Déficit moteur modéré } \\
\text { Ptosis, parésie faciale } \\
\text { Pseudo-hypertrophie } \\
\text { des mollets } \\
\text { Cardiomyopathie } \\
\text { dilatée sévère et précoce } \\
\text { Évolution rapide. Décès } \\
<20 \text { ans }\end{array}$ & $\begin{array}{l}\text { Aspect dystrophique } \\
\text { Minicores } \\
\text { Dépôts basophiles en } \\
\text { forme d'étoile. }\end{array}$ & $\begin{array}{l}\text { - Deux familles consanguines } \\
\text { portant un variant frameshift } \\
\text { homozygote dans les exons } 358 \text { et } \\
360 \text { [15] }\end{array}$ \\
\hline $\begin{array}{l}\text { Myopathie } \\
\text { congénitale à } \\
\text { multiminicores avec } \\
\text { cardiomyopathie }\end{array}$ & AR & $<20$ ans & $\begin{array}{l}\text { Déficit à prédominance } \\
\text { axiale } \\
\text { Rétractions diffuses avec } \\
\text { "rigid spine" }\end{array}$ & $\begin{array}{l}\text { Minicores } \\
\text { Dépôts basophiles en } \\
\text { forme d'étoile }\end{array}$ & $\begin{array}{l}\text { - Deux familles avec } 2 \text { variants } \\
\text { dans les exons } 359 \text { et } 360 \\
\text { entrainant un décalage du cadre de } \\
\text { lecture [16] } \\
\text { - Deux familles avec un variant } \\
\text { tronquant dans l'exon } 359 \text { associé } \\
\text { avec un autre variant faux-sens } \\
\text { dans une autre région (exons } 28 \text { et } \\
\text { 316) [16] }\end{array}$ \\
\hline $\begin{array}{l}\text { Myopathie } \\
\text { "centronucléaire" }\end{array}$ & AR & $<20$ ans & $\begin{array}{l}\text { Déficit axial et proximal } \\
\text { modéré } \\
\text { Parésie faciale } \\
\text { Syndrome restrictif } \\
\text { Absence de } \\
\text { cardiomyopathie }\end{array}$ & $\begin{array}{l}\text { Noyaux centraux dans la } \\
\text { plupart des fibres } \\
\text { musculaires } \\
\text { Minicores }\end{array}$ & $\begin{array}{l}\text { - Variants frameshift, non-sens ou } \\
\text { d'épissage [17] } \\
\text { - Distribution hétérogène sur } \\
\text { différentes régions du gène [17] }\end{array}$ \\
\hline $\begin{array}{l}\text { Dystrophie musculaire } \\
\text { proximale }\end{array}$ & AR & Adulte & $\begin{array}{l}\text { Déficit proximal moins } \\
\text { sévère que LGMD2J }\end{array}$ & Aspect dystrophique & $\begin{array}{l}\text { - Une famille italienne et une autre } \\
\text { finlandaise portant le variant } \\
\text { FINmaj avec un autre variant } \\
\text { faux-sens dans les exons } 305 \text { et } \\
340 \text { respectivement [22] [9] } \\
\text { - Une famille roumaine avec deux } \\
\text { variants faux-sens [23] }\end{array}$ \\
\hline $\begin{array}{l}\text { LGMD avec } \\
\text { cardiomyopathie }\end{array}$ & $A D$ & Adulte & $\begin{array}{l}\text { Cardiomyopathie sévère } \\
\text { et LGMD modéré }\end{array}$ & Minicores & $\begin{array}{l}\text { - Variant génétique bande A, trois } \\
\text { familles françaises }\end{array}$ \\
\hline $\begin{array}{l}\text { Myopathie distale des } \\
\text { membres inférieurs à } \\
\text { prédominance } \\
\text { postérieure }\end{array}$ & $A D$ & Adulte & $\begin{array}{l}\text { Déficit distal avec } \\
\text { évolution proximale } \\
\text { Atteinte des mollets }\end{array}$ & $\begin{array}{l}\text { Noyaux centraux } \\
\text { Minicores }\end{array}$ & $\begin{array}{l}\text { - Deux familles françaises } \\
\text { porteuses d'une délétion des } \\
\text { exons } 11 \text { à } 18 \text { [24] (manuscrit en } \\
\text { cours de préparation) }\end{array}$ \\
\hline
\end{tabular}

Tableau I

Phénotypes cliniques, histologiques et variants génétiques identifiées dans les différentes formes de titinopathies. 


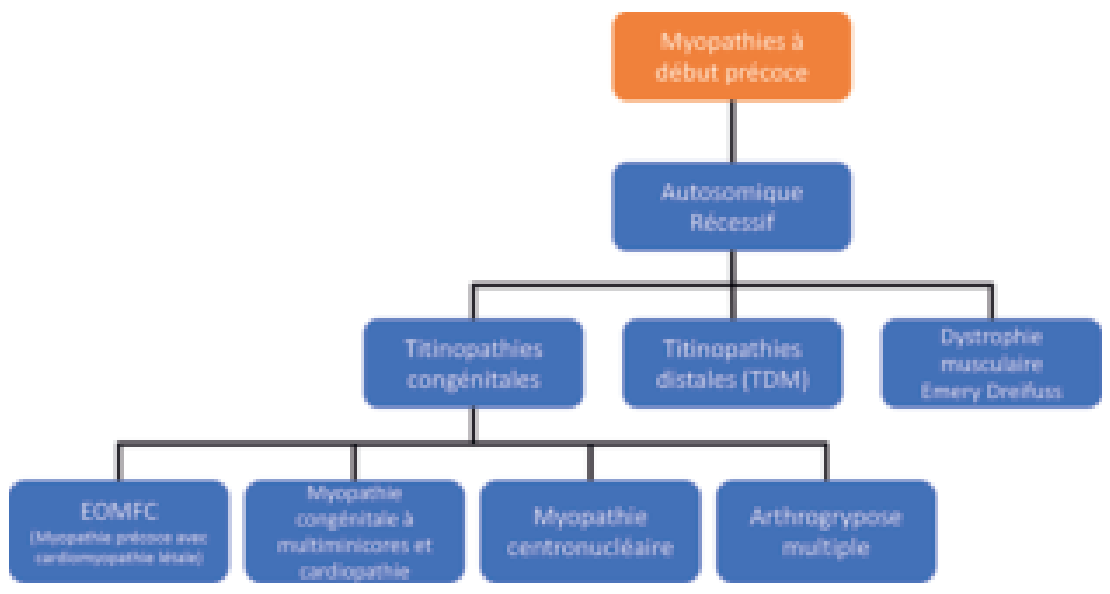

Figure 1

Synthèse des phénotypes identifiés à apparition précoce.

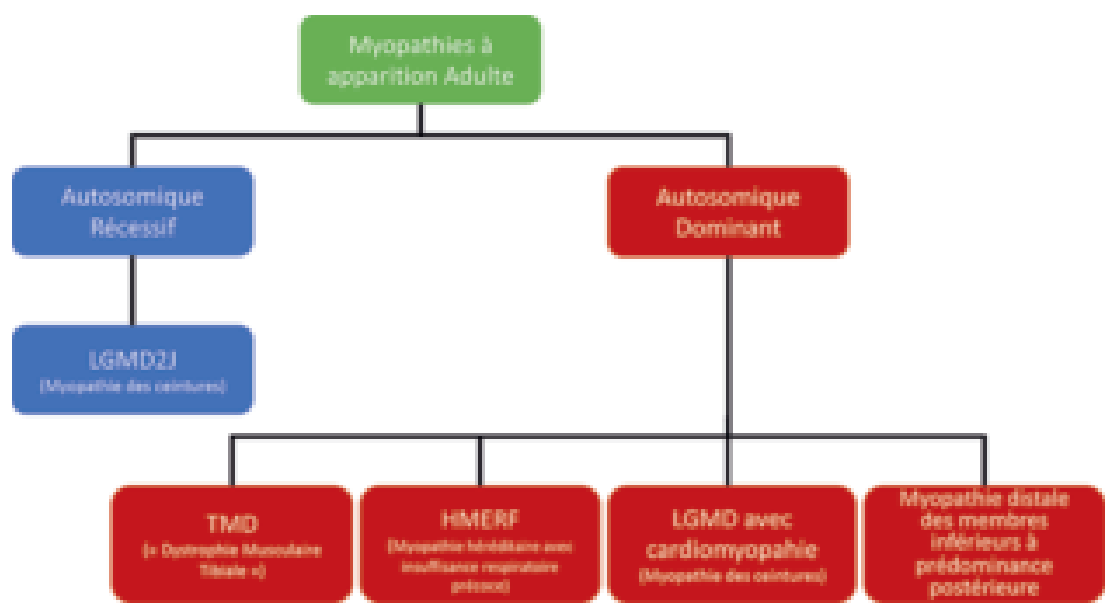

Figure 2

Synthèse des phénotypes identifiés à apparition plus tardive.

gène sans qu'une relation génotype-phénotype claire n'ait été identifiée. Concernant le type de variant, $1 / 3$ étaient de type frameshift, $1 / 3$ des nonsens et $1 / 3$ des variants d'épissage [20].

\section{Phénotypes récemment identifiés}

Myopathie distale des membres inférieurs à prédominance postérieure

Deux familles avec un phénotype de myopathie distale à prédominance postérieure ont récemment été identifiées (manuscrit en cours de préparation). Au début de la maladie, ce nouveau phénotype distal implique principalement les muscles des mollets. Au cours de l'évolution de la maladie, le déficit s'étend au niveau des jambiers antérieurs et de la partie postérieure des cuisses. La biopsie musculaire montre une inégalité de la taille des fibres, de nombreuses centralisations nucléaires et des aspects de minicores confirmés à la microscopie électronique. Le taux de CPK est normal. Les données de séquençage ont révélé une délétion hétérozygote des exons 11 à 18 induisant un décalage du cadre de lecture et l'apparition d'un codon stop prématuré. Une particularité de ce phénotype est son mode de transmission autosomique dominant. Il est intéressant de noter qu'il n'y a pas d'atteinte cardiaque chez ces patients, à l'inverse de ce que l'on observe dans les principaux phénotypes de titinopathies dominantes associées a des variants tronquants.

Myopathie à transmission autosomique dominante à atteinte cardiaque et squelettique (LGMD avec cardiomyopathie)

Un phénotype non décrit dans la littérature commence à émerger à partir des données remontant des centres de référence neuromusculaire. Ces patients présentent une atteinte cardiaque de type 
cardiomyopathie dilatée associée à un déficit musculaire squelettique modéré et un phénotype de type myopathie des ceintures (LGMD pour limb girdle muscular dystrophy). Les titinopathies du muscle squelettique sont majoritairement de transmission $\mathrm{AR}$, en dehors de tableaux de TMD et HMERF. À la différence d'autres phénotypes avec atteinte musculaire cardiaque et squelettique, celui-ci est associée à un mode de transmission autosomique dominant. Les mutations identifiées chez ces patients sont localisées dans la bande A. Contrairement aux données actuelles de la littérature où les variants tronquants hétérozygotes dans la bande $\mathrm{A}$ du gène TTN sont impliqués dans des tableaux de cardiomyopathie dilatée isolée, l'existence de ces familles démontre la possibilité d'une atteinte associée du muscle squelettique. Une évaluation exhaustive et systématique du muscle squelettique dans ces cas précis serait souhaitable.

Depuis l'article des Cahiers de Myologie de 2017 documenté par Ana Ferreiro [26], de nouveaux phénotypes continuent d'apparaître dans la littérature. Néanmoins, la physiopathologie de ces myopathies reste encore un mystère pour la majorité d'entre elles. C'est pourquoi il est important de documenter de façon exhaustive les titinopathies dans le but d'améliorer leur diagnostic et permettre une meilleure interprétation clinico-biologique des variants du gène TTN chez des patients suspectés de titinopathie (voir l'article de A. Perrin et al. dans ce numéro des Cahiers de Myologie (page 9), "Une approche clinico-biologique intégrée pour interpréter la pathogénicité des variants du gène de la titine").

\section{Phenotype-genotype correlations in titinopa-} thies

\section{LIENS D'INTÉRÊT}

Les auteurs déclarent n'avoir aucun lien d'intérêt concernant les données publiées dans cet article.

\section{RÉFÉRENCES}

1. Fang HJ, Liu BP. Prevalence of TTN mutations in patients with dilated cardiomyopathy. Herz 2019 June 17. doi:10.1007/ s00059-019-4825-4.

2. Udd B, Hakamies L, Partanen J, Laulumaa V, et al. Tibial muscular dystrophy: late adult-onset distal myopathy In 66 Finnish patients. Arch Neurol 1993 ; 50 : 604-8

3. Hackman P, Vihola A, Haravuori H, et al. Tibial muscular dystrophy is a titinopathy caused by mutations in TTN, the gene encoding the giant skeletal-muscle protein titin. Am J Hum Genet $2002 ; 71: 492-500$

4. Pollazzon M, Suominen T, Penttilä S, et al. The first Italian family with tibial muscular dystrophy caused by a novel titin mutation. J Neurol 2010 ; 257 : 575-9.

5. Van den Bergh PYK, Bouquiaux O, Verellen C, Marchand S, Richard I, Hackman P, Udd B. Tibial muscular dystrophy in a Belgian family. Ann Neurol 2003 ; 54 : 248-51.
6. Hackman P, Marchand S, Sarparanta J, et al. Truncating mutations in C-terminal titin may cause more severe tibial muscular dystrophy (TMD). Neuromuscul Disord 2008 ; 18 : 922-8.

7. Pfeffer G, Barresi R, Wilson IJ, et al. A. P.15.7 A founder mutation in the titin gene is a common cause of myofibrillar myopathy with early respiratory failure. Neuromuscul Disord 2013 ; $23: 280$.

8. Palmio J, Leonard-Louis S, Sacconi S, et al. Expanding the importance of HMERF titinopathy: new mutations and clinical aspects. J Neurol 2019 ; 266 : 680-90.

9. Evilä A, Vihola A, Sarparanta J, et al. Atypical phenotypes in titinopathies explained by second titin mutations. Ann Neurol $2014 ; 75: 230-40$

10. Perić S, Glumac JN, Töpf A, et al. A novel recessive TTN founder variant is a common cause of distal myopathy in the Serbian population. Eur J Hum Genet 2017 ; 25 : 572-81.

11. Udd B, Rapola J, Nokelainen P, Arikawa E, Somer H. Nonvacuolar myopathy in a large family with both late adult onset distal myopathy and severe proximal muscular dystrophy. J Neurol Sci $1992 ; 113$ : 214-21.

12. Pénisson-Besnier I, Hackman P, Suominen T, Sarparanta J, Huovinen S, Richard-Crémieux I, Udd B. Myopathies caused by homozygous titin mutations: Limb-girdle muscular dystrophy $2 \mathrm{~J}$ and variations of phenotype. $J$ Neurol Neurosurg Psychiatry $2010 ; 81: 1200-2$.

13. Zheng W, Chen H, Deng X, Yuan L, Yang Y, Song Z, Yang $\mathrm{Z}$, Wu Y, Deng $\mathrm{H}$. Identification of a novel mutation in the titin gene in a Chinese family with limb-girdle muscular dystrophy $2 \mathrm{~J}$. Mol Neurobiol 2016 ; 53 : 5097-102

14. De Cid R, Ben Yaou R, Roudaut C, et al. A new titinopathy: Childhood-juvenile onset Emery-Dreifuss-like phenotype without cardiomyopathy. Neurology 2015 ; 85 : 2126-35.

15. Carmignac V, Salih MAM, Quijano-Roy S, et al. C-terminal titin deletions cause a novel early-onset myopathy with fatal cardiomyopathy. Ann Neurol 2007 ; 61 : 340-51.

16. Chauveau C, Bonnemann CG, Julien C, et al. Recessive TTN truncating mutations define novel forms of core myopathy with heart disease. Hum Mol Genet 2014 ;23 : 980-91.

17. Ceyhan-Birsoy O, Agrawal PB, Hidalgo C, et al. Recessive truncating titin gene, TTN, mutations presenting as centronuclear myopathy. Neurology 2013 ; 81 : 1205-14.

18. Fernández-Marmiesse A, Carrascosa-Romero MC, Alfaro Ponce $\mathrm{B}$, et al. Homozygous truncating mutation in prenatally expressed skeletal isoform of TTN gene results in arthrogryposis multiplex congenita and myopathy without cardiac involvement. Neuromuscul Disord 2017 ; 27 : 188-92.

19. Savarese M, Sarparanta J, Vihola A, Udd B, Hackman P. Increasing role of titin mutations in neuromuscular disorders. $J$ Neuromuscul Dis 2016 ; 3 : 293-308.

20. Oates EC, Jones KJ, Donkervoort S, et al. Congenital Titinopathy: Comprehensive characterization and pathogenic insights. Ann Neurol 2018; 83 : 1105-24.

21. Udd B, Vihola A, Sarparanta J, Richard I, Hackman P. Titinopathies and extension of the M-line mutation phenotype beyond distal myopathy and LGMD2J. Neurology 2005 ; 64 : 636-42. 22. Evila A, Udd B, Hackman P. A targeted next-generation sequencing panel for diagnostic use in primary myopathies. $\mathrm{Neu}$ romuscul Disord $2014 ; 24: 800$.

23. Dabby R, Sadeh M, Hilton-Jones D, Plotz P, Hackman P, Vihola A, Udd B, Leshinsky-Silver E. Adult onset limb-girdle muscular dystrophy. A recessive titinopathy masquerading as myositis. $J$ Neurol Sci 2015 ; 351 : 120-3.

24. Zenagui $\mathrm{R}$, Lacourt $\mathrm{D}$, Pegeot $\mathrm{H}$, et al. A reliable targeted next-generation sequencing strategy for diagnosis of myopathies and muscular dystrophies, especially for the giant titin and nebulin genes. J Mol Diagn 2018; 20 : 533-49.

25. Savarese M, Maggi L, Vihola A, et al. Interpreting genetic variants in titin in patients with muscle disorders. JAMA Neurol 2018 ; 75 : 557-65.

26. Ferreiro A, Urtizberea JA. Pathologies musculaires liées à la titine : un domaine en émergence. Med Sci (Paris) 2017 ; 33 (hors série 1 - Les Cahiers de Myologie) : 16-26. 